\title{
A Note on Haselgrove's Method for Numerical Integration
}

\section{By Masaaki Sugihara and Kazuo Murota}

Abstract. An alternative set of weights is proposed for Niederreiter's generalization of Haselgrove's method for numerical integration.

1. In 1961, C. B. Haselgrove [1] introduced effective $d$-dimensional cubature formulas for a certain class of integrand functions over $G_{d}\left(\equiv[0,1]^{d}\right)$ which were generalized by $\mathrm{H}$. Niederreiter [2], [3]. But the generalized cubatures are not necessarily easy to handle. Here we shall propose new cubature formulas which are more convenient to use and have the same order of accuracy as Niederreiter's.

2. First, to make the above statements exact, we introduce the definition and notation.

Let $P_{d}^{k}(C)$ denote the family of real valued functions defined over $G_{d}$, and periodically extended over $\mathbf{R}^{d}$, which satisfy the following two conditions:

Condition 1 . Function $f(\mathbf{x})$ can be expanded into an absolutely convergent multiple Fourier series

$$
f(\mathbf{x})=\sum_{\mathbf{h}} c_{\mathbf{h}} \exp (2 \pi i\langle\mathbf{h}, \mathbf{x}\rangle),
$$

where $\langle$,$\rangle is the inner product, and \Sigma_{\mathbf{h}}$ denotes the summation of $\mathbf{h}$ over $\mathbf{Z}^{d}(\mathbf{Z}$ : integers).

Condition 2. All the Fourier coefficients $c_{\mathbf{h}}$ of $f(\mathbf{x})$ with $\mathbf{h} \neq \mathbf{0}$ satisfy the inequalities

$$
\left|c_{\mathbf{h}}\right| \leqslant C(r(\mathbf{h}))^{-k},
$$

where $r(\mathbf{h})$ designates $\Pi_{j=1}^{d} \max \left(1,\left|h_{j}\right|\right)$.

Now with this preparation, we quote the theorem by $\mathrm{H}$. Niederreiter [2].

THEOREM 1. Let $k$ be a positive integer, and let $\alpha_{1}, \ldots, \alpha_{d}(d \geqslant 1)$ be algebraic numbers such that $1, \alpha_{1}, \ldots, \alpha_{d}$ are linearly independent over the rationals. Let weights $a_{N, j}^{(k)}$ be determined from the polynomial identity:

$$
\left(\sum_{j=0}^{N-1} z^{j}\right)^{k}=\sum_{j=0}^{k(N-1)} a_{N, j}^{(k)} z^{j} .
$$

Received August 7, 1981; revised December 16, 1981.

1980 Mathematics Subject Classification. Primary 65D30. 
Then we have

$$
\int_{G_{d}} f(\mathbf{x}) d \mathbf{x}-N^{-k} \sum_{j=0}^{k(N-1)} a_{N, j}^{(k)} f\left(j \alpha_{1}, \ldots, j \alpha_{d}\right)=O\left(N^{-k}\right)
$$

for every $f \in P_{d}^{k+\lambda}(C)$ with arbitrary $\lambda>0$.

Moreover, $\mathrm{H}$. Niederreiter gave a way to evaluate the summation in the left-hand side of (4) without direct computation of $a_{N, j}^{(k)}$. However, his method requires a huge number, $O\left(N^{k}\right)$, of additions. Hence it is evident that his method is not suitable for practical computation. On the other hand, direct computation of $a_{N, j}^{(k)}$ must be performed by the formula

$$
a_{N, j}^{(k)}=\sum_{m=0}^{[j / N]}(-1)^{m}\left(\begin{array}{c}
k \\
m
\end{array}\right)\left(\begin{array}{c}
j-N m+k-1 \\
k-1
\end{array}\right),
$$

which is not convenient for practical use, either. Here we will propose an alternative set of weights which is easily computable from a simple function as below. Precisely speaking, we have the next theorem.

THEOREM 2. Let

$$
w^{(k)}(x)=\frac{(2 k+1) !}{k ! k !} x^{k}(1-x)^{k}
$$

where $(2 k+1) ! /(k ! k !)$ is a normalizing constant so that $\int_{0}^{1} w^{(k)}(x) d x=1$. Then under the same assumptions as in Theorem 1 , we have

$$
\int_{G_{d}} f(\mathbf{x}) d \mathbf{x}-N^{-1} \sum_{j=0}^{N-1} w^{(k)}\left(\frac{j}{N}\right) f\left(j \alpha_{1}, \ldots, j \alpha_{d}\right)=O\left(N^{-k}\right)
$$

for every $f \in P_{d}^{k+\lambda}(C)$ with arbitrary $\lambda>0$.

Proof. In the first place, the absolute value of the error is estimated in terms of the Fourier coefficients of $f(\mathbf{x})$. Noting the identity

$$
c_{\mathbf{0}}=\int_{G_{d}} f(\mathbf{x}) d \mathbf{x}
$$

we have from (1)

$$
\begin{aligned}
\mid \text { Error } \mid= & \left|N^{-1} \sum_{j=0}^{N-1} w^{(k)}\left(\frac{j}{N}\right) f\left(j \alpha_{1}, \ldots, j \boldsymbol{\alpha}_{d}\right)-\int_{G_{d}} f(x) d x\right| \\
= & \left|N^{-1} \sum_{j=0}^{N-1} w^{(k)}\left(\frac{j}{N}\right)\left(\sum_{\mathbf{h}} c_{\mathbf{h}} \exp (2 \pi i j\langle\boldsymbol{\alpha}, \mathbf{h}\rangle)\right)-c_{\mathbf{0}}\right| \\
\leqslant & \left|c_{\mathbf{0}}\right|\left|N^{-1} \sum_{j=0}^{N-1} w^{(k)}\left(\frac{j}{N}\right)-1\right| \\
& +\left|\sum_{\mathbf{h} \neq \mathbf{0}} c_{\mathbf{h}}\left(N^{-1} \sum_{j=0}^{N-1} w^{(k)}\left(\frac{j}{N}\right) \exp (2 \pi i j\langle\mathbf{\alpha}, \mathbf{h}\rangle)\right)\right|,
\end{aligned}
$$

where $\boldsymbol{\alpha}=\left(\alpha_{1}, \ldots, \alpha_{d}\right)$, and $\Sigma_{\mathbf{h} \neq \mathbf{0}}$ denotes the summation of $\mathbf{h}$ over $\mathbf{Z}^{d}$ except $\mathbf{0}$. For 
brevity, we define here the function

$$
K_{N}^{(k)}(\theta)=N^{-1} \sum_{j=0}^{N-1} w^{(k)}\left(\frac{j}{N}\right) \exp (2 \pi i j \theta) .
$$

Then the expression (9) is simply rewritten as

$$
\mid \text { Error }|\leqslant| c_{\mathbf{0}}|| K_{N}^{(k)}(0)-1|+| \sum_{\mathbf{h} \neq \mathbf{0}} c_{\mathbf{h}} K_{N}^{(k)}(\langle\boldsymbol{\alpha}, \mathbf{h}\rangle) \mid \text {. }
$$

This inequality for the error indicates that it is essential to estimate this function $K_{N}^{(k)}(\theta)$ for $\theta=0$ and $\theta$ irrational.

Next we proceed to evaluate $K_{N}^{(k)}(\theta)$. Poisson's summation formula [8] yields the identity

$$
\begin{aligned}
K_{N}^{(k)}(\theta) & =\lim _{\nu \rightarrow \infty} \sum_{l=-\nu}^{\nu} N^{-1} \int_{0}^{N} w^{(k)}\left(\frac{x}{N}\right) \exp (2 \pi i \theta x-2 \pi i l x) d x \\
& =\lim _{\nu \rightarrow \infty} \sum_{l=-\nu}^{\nu} \int_{0}^{l} w^{(k)}(y) \exp (2 \pi i N y(\theta-l)) d y .
\end{aligned}
$$

On the other hand, repeated partial integration leads to

$$
\begin{aligned}
& \left|\int_{0}^{1} w^{(k)}(y) \exp (2 \pi i N y(\theta-l)) d y\right| \\
& \quad \leqslant \frac{1}{|2 \pi N(\theta-l)|^{k}} \int_{0}^{1}\left|D^{k} w^{(k)}(y)\right| d y \quad \text { for } \theta \neq l,
\end{aligned}
$$

where $D^{k}=d^{k} / d x^{k}$. Here we notice that $D^{k} w^{(k)}(x)$ is equal to

$$
((2 k+1) ! / k !) P_{k}(2 x-1),
$$

where $P_{k}$ is the Legendre polynomial over $[-1,1]$ of degree $k$. By the property that $\sup _{-1 \leqslant x \leqslant 1}\left|P_{k}(x)\right| \leqslant 1$, we obtain

(12) $\left|\int_{0}^{l} w^{(k)}(y) \exp (2 \pi i N y(\theta-l)) d y\right| \leqslant \frac{(2 k+1) !}{k !} \frac{1}{|2 \pi N(\theta-l)|^{k}} \quad$ for $\theta \neq l$.

The combination of (11) with (12) gives the estimate for $K_{N}^{(k)}(\theta)$ :

$$
\begin{aligned}
&\left|K_{N}^{(k)}(\theta)\right| \leqslant \frac{(2 k+1) !}{k !} \frac{1}{(2 \pi N)^{k}}\left(\frac{2}{\|\theta\|^{k}}+2 \sum_{l=1}^{\infty} \frac{1}{l^{k}}\right) \\
& \leqslant \frac{(2 k+1) !}{k !} \frac{2(1+\zeta(k))}{(2 \pi)^{k}} \frac{1}{N^{k}\|\theta\|^{k}} \quad \text { for } \theta \text { irrational, } \\
&\left|K_{N}^{(k)}(0)-1\right| \leqslant \frac{(2 k+1) !}{k !} \frac{2 \zeta(k)}{(2 \pi)^{k}} \frac{1}{N^{k}} \quad \text { for } \theta=0,
\end{aligned}
$$

where $\zeta$ is Riemann's zeta function and $\|x\|$ is the distance from $x$ to the nearest integer.

Consequently, substituting these inequalities (13), (14) into (10), we reach

$$
\mid \text { Error } \mid \leqslant \frac{1}{N^{k}} \frac{2 \cdot(2 k+1) !}{(2 \pi)^{k} k !}\left(\left|c_{\mathbf{0}}\right| \zeta(k)+(1+\zeta(k)) \sum_{\mathbf{h} \neq \mathbf{0}}\left|c_{\mathbf{h}}\right| \frac{1}{\|\langle\boldsymbol{\alpha}, \mathbf{h}\rangle\|^{k}}\right) .
$$


The rest of the proof is the same as that of Niederreiter's theorem. Q.E.D.

Remark. The particular form (6) of the weight function is not essential for Theorem 2 to hold. In effect, it is sufficient that the weight function $w^{(k)}(x)$ satisfies the following conditions:

(1) $\int_{0}^{1} w^{(k)}(x) d x=1$,

(2) $w^{(k)}(x)$ is $k$ times continuously differentiable and $w^{(k)}(0)=w^{(k)}(1), D w^{(k)}(0)$ $=D w^{(k)}(1), \ldots, D^{k-1} w^{(k)}(0)=D^{k-1} w^{(k)}(1)$, where $D^{k}=d^{k} / d x^{k}$.

Remark. The restriction of the integrand function in Theorem 2 is not serious from the practical point of view; an appropriate polynomial transformation of the integration variables $x_{j}=\phi\left(t_{j}\right)(j=1, \ldots, d)$, where

$$
\phi(t)=c \int_{0}^{t}(s(1-s))^{\alpha} d s
$$

usually brings the integrand function into the class $P_{d}^{k+\lambda}(C)$ [3] as will be done in the numerical experiment in Section 3. Moreover, for a certain class of integrations, the IMT-type transformation of integration variables [4], [5], [6] transforms the integrand function into the one with Fourier coefficients $c_{h}$ satisfying

$$
\left|c_{\mathrm{h}}\right| \leqslant C \exp \left(-A(\delta) \sum_{j=1}^{d}\left|h_{j}\right|^{1-\delta}\right) \quad \text { with } A(\delta)>0 \text { and arbitrary } \delta>0 \text {. }
$$

Hence, considering the fact that the transformed integrand function belongs to $P_{d}^{\lambda}(C(\lambda))$ for all $\lambda$, we may expect that we can do much better by choosing a suitable weight function. In fact, with the weight function of

(i) Gaussian-type

$$
w_{N}(x)=\sqrt{\frac{B}{\pi N^{2-\alpha}}} \exp \left(-B N^{\alpha}\left(x-\frac{1}{2}\right)^{2}\right) \quad\left(\alpha=\frac{1}{d+1}, B>0\right),
$$

or

(ii) IMT-type [4], [5], [6],

$$
w(x)=\frac{d}{d x}\left(\frac{1}{2} \tanh \left(\frac{\pi}{2} \sinh \frac{\pi}{4}\left(-\frac{1}{x}+\frac{1}{1-x}\right)\right)\right),
$$

the asymptotic accuracy of

$$
O\left(\exp \left(-A^{\prime}(\varepsilon) N^{1 /(d+1)-\varepsilon}\right)\right) \quad \text { with } A^{\prime}(\varepsilon)>0 \text { and arbitrary } \varepsilon>0,
$$

is attained for the transformed integrand function. The result will not be obtained until the weight function as in Theorem 2 is introduced from a viewpoint different from Niederreiter's Theorem 1.

3. In Section 2, we have proved that the theoretical order of accuracy of the proposed cubature with $w^{(k)}(x)$ is equal to that of Niederreiter's with $a_{N, j}^{(k)}$. To compare the actual performances, we present in this part the numerical results for a 4-dimensional problem. To be specific, the two methods are applied to the numerical evaluation of

$$
\int_{G_{4}} f(\mathbf{x}) d \mathbf{x}(=1), \quad f(\mathbf{x})=\prod_{j=1}^{4} \frac{0.11}{\left(0.1+x_{j}\right)^{2}}
$$


which was used in [7] as an example illustrating that the original Haselgrove's method does not always give a significant solution. We transform the integrand function $f$ into

$$
f\left(\phi\left(x_{1}\right), \ldots, \phi\left(x_{4}\right)\right) \cdot \prod_{j=1}^{4} \phi^{\prime}\left(x_{j}\right), \quad \phi(x)=\int_{0}^{x} 2772 t^{5}(1-t)^{5} d t,
$$

in order that the transformed integrand function may belong to $P_{4}^{5}(C)$. We adopt

$$
2 \cos \frac{2 \pi}{11}, 2 \cos \frac{4 \pi}{11}, 2 \cos \frac{6 \pi}{11}, 2 \cos \frac{8 \pi}{11},
$$

for $\alpha_{1}, \alpha_{2}, \alpha_{3}, \alpha_{4}$, following the recommendation by many authors.

Among the numerical experiments with various weights, we give only the result for Niederreiter's method with $a_{N, j}^{(4)}$ and for the proposed one with $w^{(4)}(x)$ (Figure 1). Note that these two methods have the same order of accuracy and that when the same number of points are used in (4) and (7), the $N$ in (2) is $k$ times the $N$ in (4). The graph in Figure 1 indicates that there may be nothing to choose between the two. Also in other cases, not shown here, the proposed method competes with Niederreiter's.

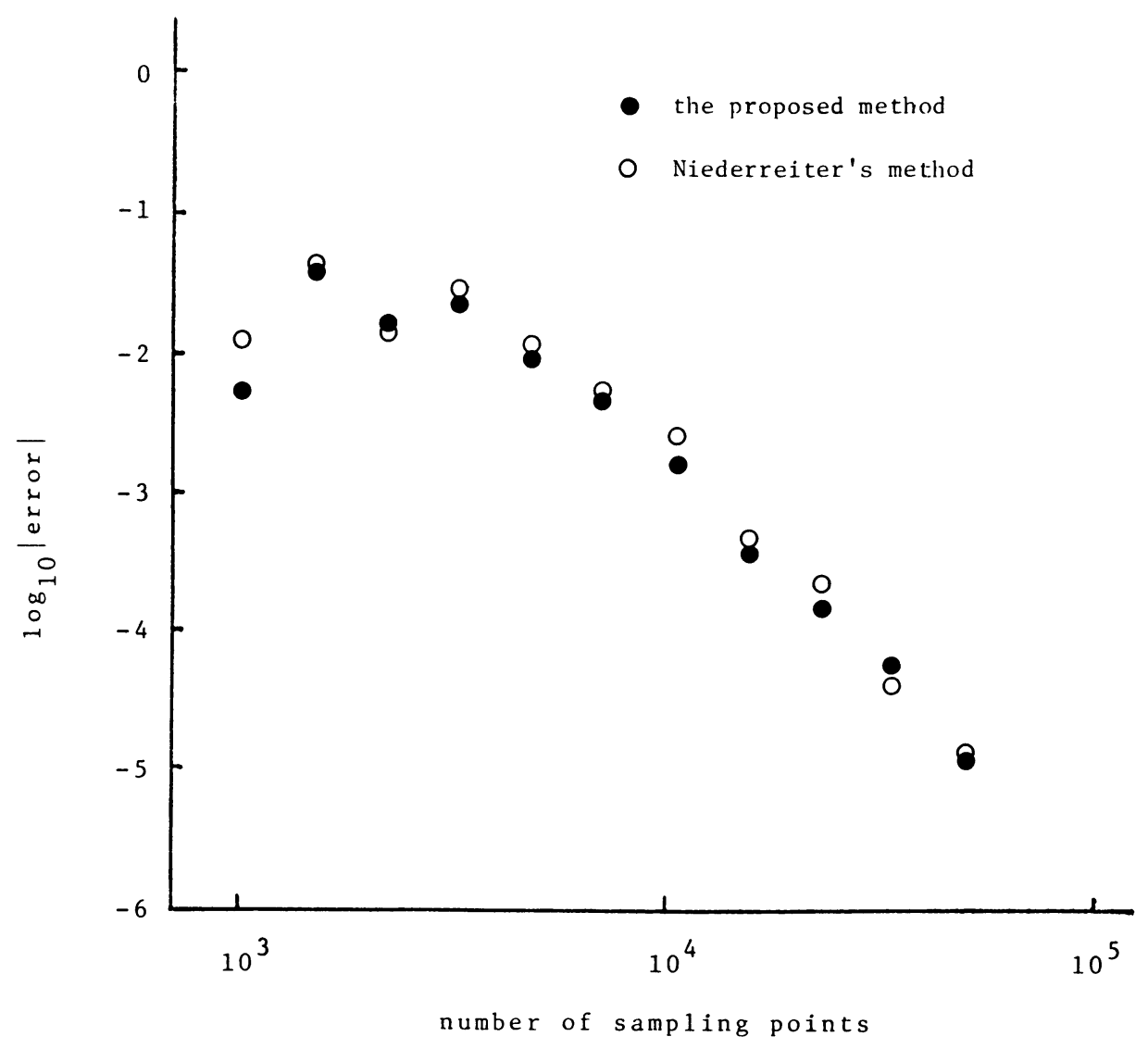

FIGURE 1

Comparison of the proposed method ( with $w^{(4)}$ ) with Niederreiter's method (with $a_{N, j}^{(4)}$ ) for a 4-dimensional problem 
4. It may be concluded that the theoretical order of accuracy of the proposed cubatures is the same as that of Niederreiter's, and that in actual experiments there is little difference between the two. Therefore in view of the ease in practice, the proposed method is recommended rather than Niederreiter's.

Acknowledgement. The authors wish to express their gratitude to the referee for his kind comments.

Department of Mathematical Engineering and Instrumentation Physics

Faculty of Engineering

University of Tokyo

Bunkyo-ku, Hongo

Tokyo 113, Japan

1. C. B. HaSelgrove, “A method for numerical integration," Math. Comp., v. 15, 1961, pp. 323-337.

2. H. NIEDERREITER, "Application of diophantine approximations to numerical integration," Diophantine Approximation and Its Applications (C. F. Osgood, ed.), Academic Press, New York, 1973, pp. 129-199.

3. H. NieDerReiter, "Quasi-Monte Carlo methods and pseudo-random numbers," Bull. Amer. Math. Soc., v. 84, 1978, pp. 957-1041.

4. P. J. Davis \& P. Rabinowitz, Methods of Numerical Integration, Academic Press, New York, 1975.

5. M. MORI, "An IMT-type double exponential formula for numerical integration," Publ. Res. Inst. Math. Sci., Kyoto Univ., v. 14, 1978, pp. 713-729.

6. K. MUROTA \& M. IRI, "Parameter tuning and repeated application of the IMT-type transformation in numerical quadrature," Numer. Math., v. 38, 1982, pp. 347-363.

7. T. Tsuda, "Numerical integration of functions of very many variables," Numer. Math., v. 20, 1973, pp. 377-391.

8. S. BOCHNER, Vorlesungen über Fouriersche Integrale, Akademische-Verlag, Leipzig, 1932. 